\title{
Modified Hue over Intensity Ratio Based Method for Shadow Detection and Removal in Arial Images
}

\author{
Abhishek Mishra and Bharti Chaurasia
}

\begin{abstract}
In this paper, we represent an outline to automatically identify and remove shadows from the real single image. The most significant features can be manipulated by multiple convolution deep neural networks (ConvNets) process. The required features extraction methods are imposed at the dominant boundaries in each super-pixel level of the image. The shadow masks may be predicted random model to generate a smooth image based on Bayesian formulation. These works represents a chromaticity based process for removal of desire shadows in aerial images. Hue is considered as the base variables for shadow detection. We represent modified hue over intensity ratio method for the better result.
\end{abstract}

\section{Keywords}

Cast Shadowing;

Hue over Intensity Ratio;

Hue, Saturation, and Value;

Min Depth Parameter;

Self Shadowing.

\section{INTRODUCTION}

The shadows in images or motion pictures may be affected by various undesired phenomena in the scene, due to various lighting conditions, shadowed surfaces, including objects surface etc[1]. Our vision system is very sensitive regarding shadows and us also able to track the objects along with shadows [2]. Shadows can be aided for scene interpretation, base on model shadows which may be include or ignore [3]. In some case, shadows degrade the quality of image [3]. Shadow detection is an essential pre-processing action for image enhancement [4]. For computer vision, shadows sometimes create various problems which may reduce the consistency of the picture degrade the image quality. In addition, shadows are also responsible to. Shadow removal is also an important criterion for image enhancement algorithm. Different image enhancement algorithms are available [5][6]. High-resolution satellite images contain a huge amount of information. Shadows in such images generate real problems in classifying and extracting the required information. Although signals recorded in shadow area are weak, it is still possible to recover them. Significant work is already done in shadow detection direction but, classifying shadow pixels from vegetation pixels correctly is still an issue as dark vegetation area are still misclassified as a shadow in cases[7]. Shadow pixels are classified from the index histograms by an automatic threshold identification procedure [8]. Shadow also depends on the observation degradation in the detection performance [9].

The lots of work is done on the image enhancement by various researchers, to enhancing edges as fundamental component of the images [11, 12], unstill shadows [13], objects clearness [14-20] etc. In this paper, an efficient shadow detection method is discussed to improve its performance which are confirm the results a better performance, compared with the other detection algorithms.

\section{SELF-SHADOWING}

The self-shadow is the feature of dynamic shadows only. Basically it allows to casting a shadows onto the world (Fig 1). We might be able to make it consistent for static shadows to make it less confusing. Fixing on static shadows might be difficult, it may break the GI. Moving cast shadow detection also plays an exceptionally important in content-based surveillance videos analysis. For proper analysis first exploit color information like saturation, hue, and Value color space and texture space needed to separate on moving cast shadows from its foreground. A rough shadow generate by synthesizing the above features after post processing misclassified pixels required to refine output image.

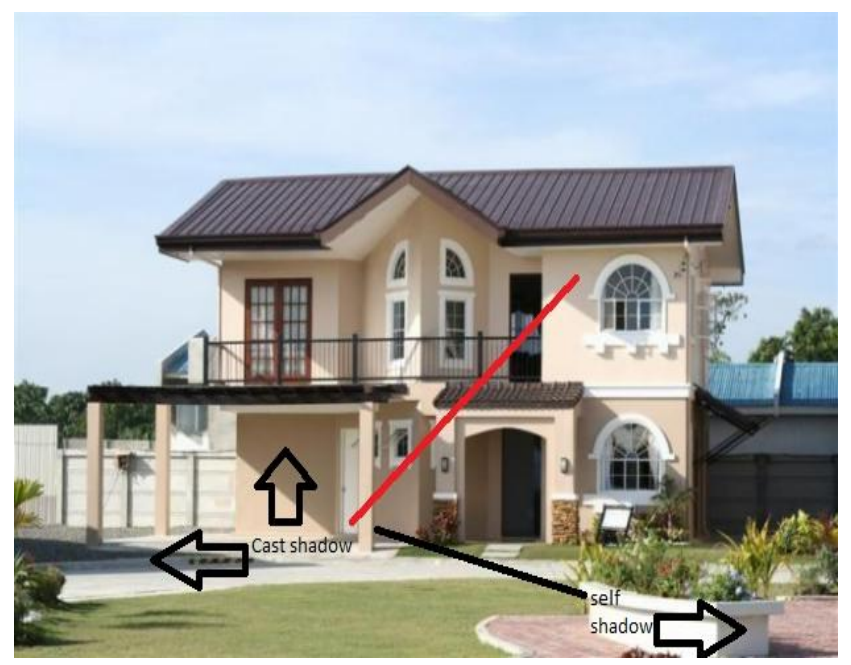

Fig1.Image showing self-shadow and cast shadow.

Self-Shadowing is a trade-offs between quality and processing speed depending on the expected result. The self- 
shadow modification also depends on spot lights ray casting (Fig 2). A spot light may be uses for shadow map alternately know as depth map to determine the shadows cast surfaces. If a surface is illuminated by a spot light as Fig 2, then the map also begins at the illuminated. Any surface belongs within this shadow area will be create shadow due to shadow map has a fixed resolution. The shadow map intersects the surface and partially overlaps with its own shadow. The Min Depth parameter keeps to a small value for better result. The renderer for the time being moves from the shadow required casting surface in the direction of the light source, prior to calculating the desired shadow map, so the target surface is no longer includes in its self shadow.

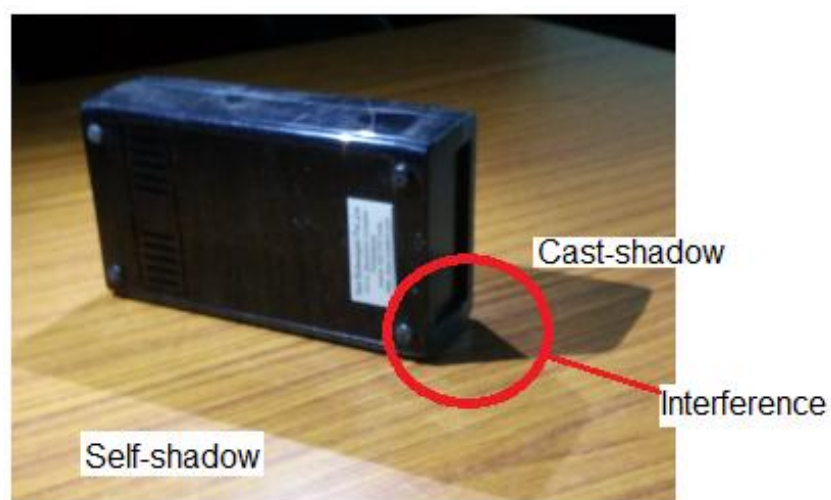

Fig 2.Spot lights ray casting. Interference between selfshadow and cast-shadow.

The Min Depth sometimes creates problem for too small a value and too large a value (Fig 3). If the Min Depth parameter is too small, the target surface partially includes in its own shadow.

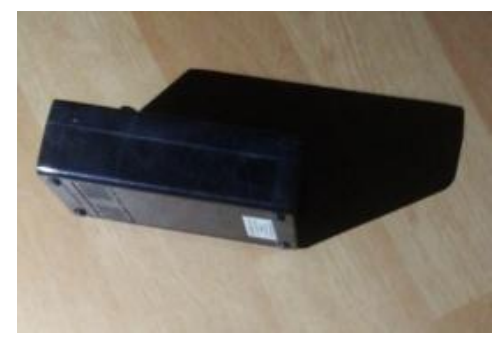

(a)

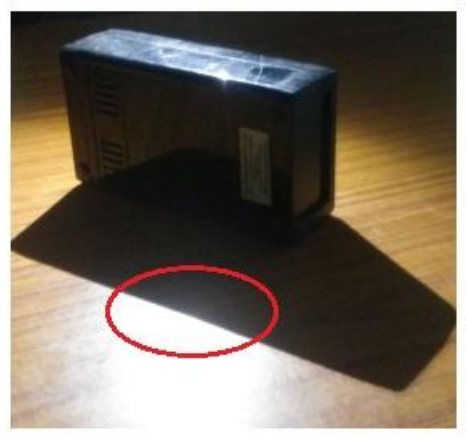

(b)

Fig 3.Min Depth surface (a) small a value and (b) large value, shadow suffer with reflected light rays (read circle).
For the case of large Min Depth value other surfaces may no longer be in shadow as per target. Min Depth value is basically in world space units; hence the magnitude of it is related with choice of scale. Using Blend Offset is a better method of solving self-shadowing problems. Whereas Min Depth moves the entire shadow casting surface an equal amount, Blend Offset moves different surface points different amounts, based on the probability that the original point on the shadow map was correct.

Self-shadowing can be even more of a problem with motion-blurred objects. The renderer calculates motion blur by simulating the shutter and exposure time of a real camera. The motion blurred object is rendered several times between the shutter's open and close times for each frame. The shadow map, however, is only calculated at the shutter mid-point. If the object is moving very fast away from the spot light, then it may appear to be entirely within its own shadow for part of the motion blur.

\section{Proposed Method}

The proposed method work on the color features of the shadow region in the image. The value of hue is high and the saturation value is low in the shadow region. The hue over saturation ratio provides the more clear characteristics for the shadow detection. The most significant features can be manipulated by multiple convolution deep neural networks (ConvNets) process. In this method, sometimes the nonshadow region, Hue is considered as the base variables for shadow detection. We represent modified hue over intensity ratio method for better result.

An efficient algorithm was presented by Tsai, by using hue over the intensity to construct the ratio mapping for identify shadows. This techniques is suitable for the identify shadows in color aerial images by help of the global thresholding value calculations. The process is very simple input image required to transform into the equivalent HSI image. The HSI is Hue ( $\mathrm{H}$ ), Saturation ( S ), Intensity ( I ) is the color space which is very efficient modeling techniques this applications[10]. Hue, saturation, and value (HSV) is mapping similar to the human eye sensitive to different colors. Luma, blue-difference Chroma, and red-difference Chroma (Y $\mathrm{CbCr}$ ) transformation and the process further continue hue, Chroma, and Value (HCV) or may using luminance, hue, and saturation (YIQ) color.

Based on HSI color model, the intensity-equivalent image $\left(\mathrm{I}_{\mathrm{e}}\right)$, the hue-equivalent $\mathrm{ImageH}_{\mathrm{e}}$ are utilized in modified ratio map by three proposed scheme

$\mathrm{I}_{\mathrm{e}}=0.26 \mathrm{R}+0.32 \mathrm{G}+0.32 \mathrm{~B}$ for reflecting surface

$\mathrm{I}_{\mathrm{e}}=0.32 \mathrm{R}+0.32 \mathrm{G}+0.26 \mathrm{Bfor}$ non-reflecting surface otherwise,

$$
\begin{aligned}
\mathrm{I}_{\mathrm{e}} & =\frac{1}{3} \mathrm{R}+\frac{1}{3} \mathrm{G}+\frac{1}{3} \mathrm{~B} \\
\mathrm{H}_{\mathrm{e}} & =\left(\tan ^{-1}\left(\frac{\mathrm{V}_{1}}{\mathrm{~V}_{2}}\right)+\pi\right) \times \frac{255}{2 \pi}
\end{aligned}
$$

where $V_{1}$ and $V_{2}$ have been explain in (1) and the value of $\mathrm{I}_{\mathrm{e}}$ and $\mathrm{H}_{\mathrm{e}}$ are $[0,255]$. The value of hue is high and the 
saturation value is low in the shadow region. The hue over saturation ratio provides the more clear characteristics for the shadow detection. The modified argument used in mapping by Eq. (3).

$$
r(x, y)=\operatorname{round}\left(\frac{H_{e}(x, y)}{I_{e}(x, y)+1.002}\right)
$$

Where $\mathrm{r}(\mathrm{x}, \mathrm{y})$ is between 0 to 255 . A spot light may be uses for shadow map alternately know as depth map to determine the shadows cast surfaces. The flowchart of the proposed modified algorithm is shown in Fig. 4. The Min Depth parameter keeps to a small value for better result. The variable min depth parameter produced better result. The problem related with Min Depth sometimes creates problems for too small a value and too large a value (Fig 3). If the Min Depth parameter is too small, the target surface partially includes in its own shadow. For the case of large Min Depth value other surfaces may no longer be in shadow as per target. Min Depth value is basically in world space units; hence the magnitude of it is related with choice of scale.

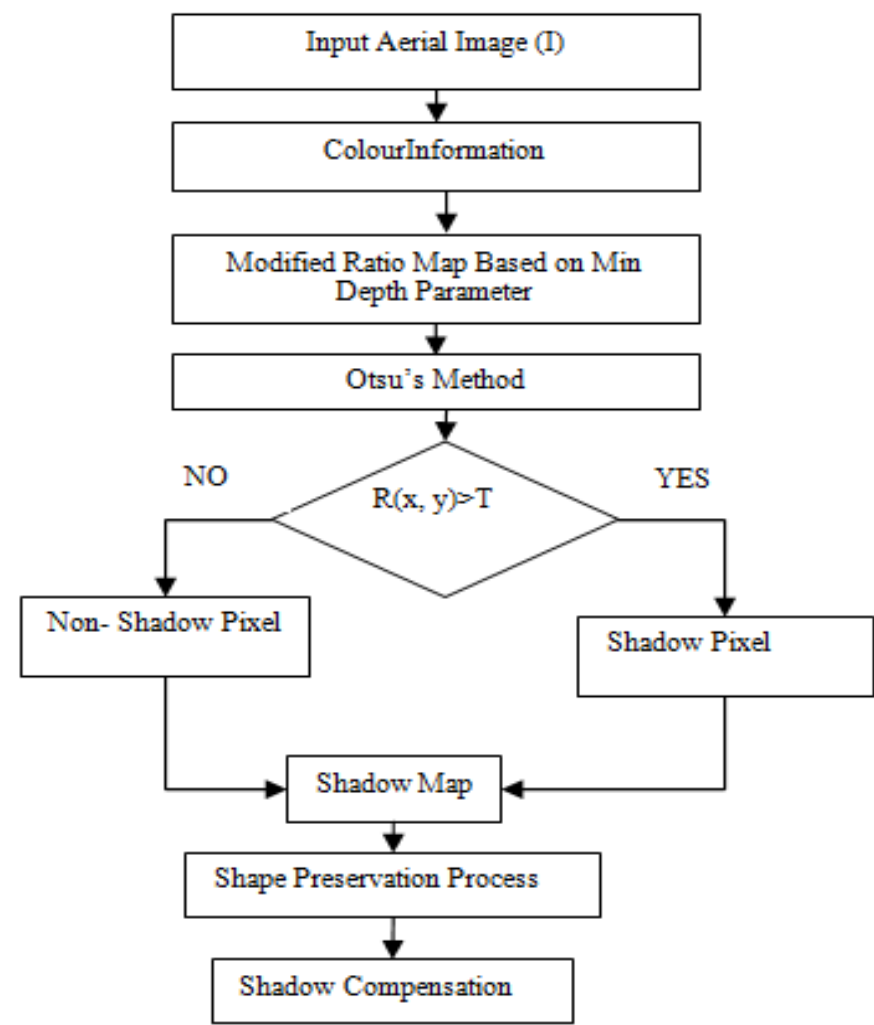

Fig.4 The flow chart of modified hue over intensity ratio based method modified Mapping algorithm.

\section{Result Analysis}

The renderer for the time being moves from the shadow required casting surface in the direction of the light source, prior to calculating the desired shadow map, so the target surface is no longer includes in its self shadow. The Colour Aerial Image Detection requires the thresholding for image segmentation. Under the same testing environments images, experimental results shown in Fig 5.

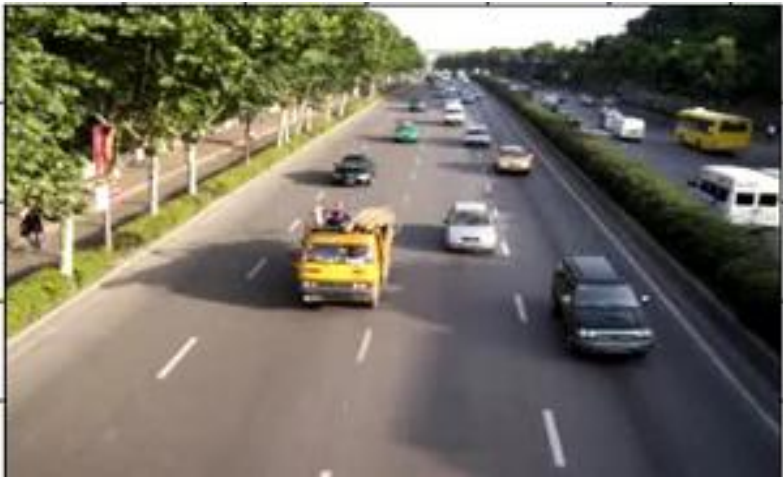

(a)

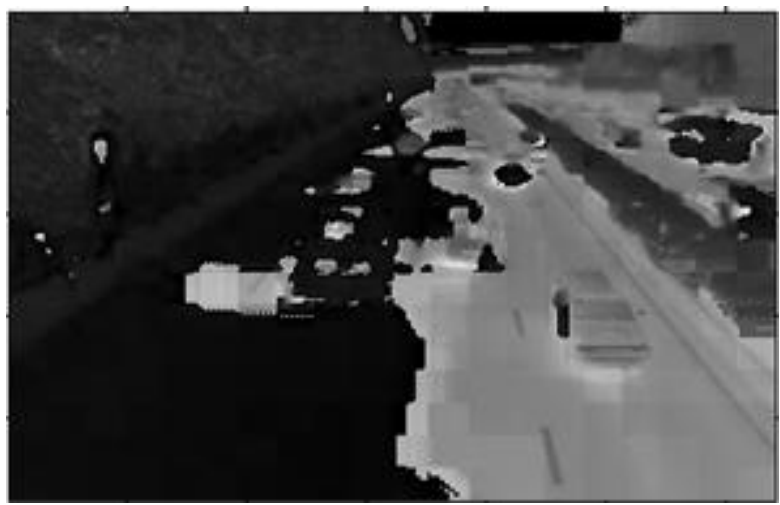

(b)

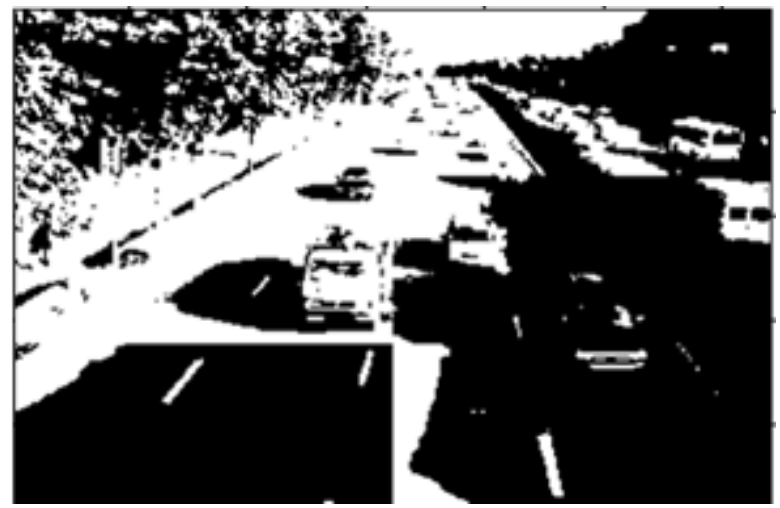

(c)

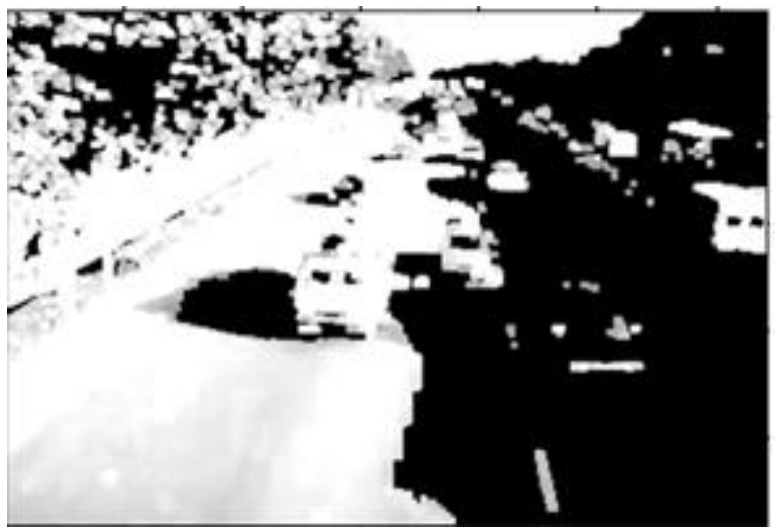

(d)

Fig 5. (a) Tested original image of highway. Comparisons between shadow detected image using (b) Tsai Model, (c) STS Method and (d) Proposed modified Hue over Intensity Ratio method.

Abhishek Mishra and Bharti Chaurasia, "Modified Hue over Intensity Ratio Based Method for Shadow Detection and Removal in Arial Images," International Journal of Advanced Engineering and Management, Vol. 2, No. 5, pp. 101-105, 2017. 


\section{A. Producer's Accuracy}

This type of accuracy contains two parameters. The parameter denotes the quantity of accurately identified true shadow pixels. Here the entire identified true shadow pixels can be used to calculate the accuracy of the shadow detection algorithm.

The first type of accuracy is the producers accuracy, which contain two parameters $\mathrm{P}_{\mathrm{S}}$ and $\mathrm{P}_{\mathrm{N}}$ and they are defined by

Producer's accuracy:

$$
\begin{aligned}
& \mathrm{P}_{\mathrm{S}}=\frac{\mathrm{TP}}{\mathrm{TP}+\mathrm{FN}} \\
& \mathrm{P}_{\mathrm{N}}=\frac{\mathrm{TN}}{\mathrm{FP}+\mathrm{TN}}
\end{aligned}
$$

TP, FP and FN are true, false and non detectable shadow pixels respectively. This is the third type of accuracy. Actually it is the combination of previous two types of accuracy produces and overall accuracy. It is used to determine the correctness percentage [15] of the algorithm. It denotes by the total number of correctly true shadow and non-shadow pixels are equal to the number of total pixel in the image.

\section{B. Overall accuracy}

The evolution matrices are used in this model to calculate the correctness of the shadow finding algorithms. There are mainly three types of accuracy these are the users accuracy, producers accuracy and the overall accuracy, these are used these evaluation. These three accuracies are based on the concept of the error matrices $[11,12]$ and terminologies [13, 14]. Now we discussed about these briefly:

Combining the accuracies $\tau$,

$$
(\tau)=\frac{\mathrm{TP}+\mathrm{TN}}{\mathrm{TP}+\mathrm{TN}+\mathrm{FP}+\mathrm{FN}}
$$

Where TP + TN denotes the number of correctly detected true shadow and non-shadow pixels; $\mathrm{TP}+\mathrm{TN}+\mathrm{FP}+\mathrm{FN}$ is equal to the number of total pixels in the image.

Table 3. Shadow Detection Accuracy for Image of Highway

\begin{tabular}{|c|c|c|c|c|c|}
\hline \multirow{2}{*}{ Method } & \multicolumn{2}{|c|}{$\begin{array}{c}\text { Producer's } \\
\text { accuracy }\end{array}$} & \multicolumn{2}{c|}{$\begin{array}{c}\text { User's } \\
\text { accuracy }\end{array}$} & $\begin{array}{c}\text { Overall } \\
\text { Accuracy }\end{array}$ \\
\cline { 2 - 6 } & $\mathrm{P}_{\mathrm{S}}(\%)$ & $\mathrm{P}_{\mathrm{N}}(\%)$ & $\begin{array}{c}\mathrm{A}_{\mathrm{S}} \\
(\%)\end{array}$ & $\begin{array}{c}\mathrm{A}_{\mathrm{N}} \\
(\%)\end{array}$ & $\tau(\%)$ \\
\hline Proposed & 94.61 & 97.84 & 81.79 & 96.59 & 89.19 \\
\hline Tsai's & 37.73 & 82.97 & 30.50 & 91.17 & 60.84 \\
\hline $\begin{array}{c}\text { Kuo-Liang } \\
\text { Chung }\end{array}$ & 37.73 & 82.79 & 40.59 & 94.32 & 67.46 \\
\hline
\end{tabular}

\section{CONCLUSION}

Here in the content we discuss about a new method for identifying the actual shadows using colour and texture. Generally misclassification arrived frequently in shadow removal algorithm which is the cause of disorder in original object shapes. Here we proposed a data-driven method to find out the most applicable description for the recognition of shadows using single image. Here in this topic we established that our framework carry out the best. There we use several numbers of applications such as image editing, development tasks etc. Also we represent a shadow removal database to identify the exact shadow and the recovered image. The proposed shadow removal is an intensity based approach which results from a more clear and understandable aerial image. Hence our present work will be performed better than the all recent shadow databases methods.

\section{REFERENCES}

[1] Nadimi, S., \& Bhanu, B. (2004). Physical models for moving shadow and object detection in video. IEEE transactions on pattern analysis and machine intelligence, 26(8), 1079-1087.

[2] Prati, A., Mikic, I., Trivedi, M. M., \& Cucchiara, R. (2003). Detecting moving shadows: algorithms and evaluation. IEEE transactions on pattern analysis and machine intelligence, 25(7), 918-923.

[3] Katre, R., \& Dodkey, N. (2017.) Rain Streaks Removal in Image via Decomposition and Visibility Feature Saturation. International Journal, 1(1), 82-85.

[4] Lee, J. S. (1980). Digital image enhancement and noise filtering by use of local statistics. IEEE transactions on pattern analysis and machine intelligence, (2), 165-168.

[5] Mishra, A., Chaurasia, B., \& Kurmi, Y. (2017). Comparative Analysis of Single Image Shadow Detection and Removal in Aerial Images. International Journal of Advanced Engineering and Management, 2(4), 86-89.

[6] Hong, L., Wan, Y., \& Jain, A. (1998). Fingerprint image enhancement: Algorithm and performance evaluation. IEEE transactions on pattern analysis and machine intelligence, 20(8), 777-789.

[7] Awad, M., Chehdi, K., \& Nasri, A. (2007). Multicomponent image segmentation using a genetic algorithm and artificial neural network. IEEE Geoscience and remote sensing letters, 4(4), 571-575.

[8] Mostafa, Y., \& Abdelhafiz, A. (2017). Shadow Identification in High Resolution Satellite Images in the Presence of Water Regions. Photogrammetric Engineering \& Remote Sensing, 83(2), 87-94.

[9] Moon, Hankyu, Rama Chellappa, and Azriel Rosenfeld. "Performance analysis of a simple vehicle detection algorithm." Image and Vision Computing 20.1 (2002): 113.

[10] Xu, H., Yang, Z., Chen, G., Liao, G., \& Tian, M. (2016). A Ground Moving Target Detection Approach Based on Shadow Feature With Multichannel HighResolution Synthetic Aperture Radar. IEEE Geoscience and Remote Sensing Letters, 13(10), 1572-1576.

[11] Ghanbari, M., Majdi, M., \& Talouki, M. (2017). Video Inpainting Using a Contour-based Method in Presence of 
[12] More than One Moving Objects. International Journal of Advanced Engineering and Management, 2(2), 37-44.

[13] Sharma, D., Kurmi, Y., \& Chaurasia, V. (2014). Formation of Super-Resolution Image: A Review. Int. Jour. of Emerging Tech. and Adv. Engg, 4(4), 218-221.

[14] Kurmi, Y., \& Chaurasia, V. (2015). An Image Fusion Approach based on Adaptive Fuzzy Logic Model with Local Level Processing. International Journal of Computer Applications, 124(1), 39-42

[15] Tiwari, S., Chauhan, K., \& Kurmi, Y. (2015). Shadow detection and compensation in aerial images using MATLAB. International Journal of Computer Applications, 119(20),1-9.

[16] Kurmi, Y., \& Chaurasia, V. (2014). Performance of Haze Removal Filter for Hazy and Noisy Images. Int. Jour. of Sci. Engg. and Tech, 3(4), 437-439.

[17] Patle, M. K., Chourasia, B. \& Kurmi. Y. (2016). "High Dynamic Range Image Analysis Through Various Tone Mapping Techniques. Int. Jour. of Comp. Appl., vol.153( 11), 14-17.

[18] Kumar, A., Chourasia, B., \& Kurmi, Y. (2017). Image Defogging by Multiscale Depth Fusion and Hybrid
Scattering Model, International Journal of Computer Applications, 155(11).

[19] W. R. Philipson, Manual of Photographic Interpretation, 2nd ed. Bethesda, MD: Amer. Soc. Photogrammetry Remote Sens., 1997.

[20] Chen, Y., Wang, J., Xu, M., He, X., \& Lu, H. (2016). A unified model sharing framework for moving object detection. Signal Processing, 124, 72-80.

[21] Mishra, P. K., \& Saroha, G. P. (2016). A study on classification for static and moving object in video surveillance system. International Journal of Image, Graphics and Signal Processing (IJIGSP), 8(5), 76-82.

\author{
Abhishek Mishra \\ setu17687@gmail.com \\ Scope college of Engineering, Bhopal, India \\ Bharti Chaurasia \\ bharti.chourasia27@gmail.com \\ Scope college of Engineering, Bhopal, India
}

\section{Editor-in-Chief: Sahadev Roy}

